\title{
PRESENCIA DEL SEXO/GÉNERO EN EL CURRÍCULUM DE LOS ESTUDIOS DE ENFERMERÍA
}

\author{
$M^{a}$ Luisa Panisello Chavarria, Inma Pastor Gosalvez, \\ $M^{a}$ Luisa Mateu Gil y $M^{a}$ Amaia Lasaga Heriz \\ Universidad Rovira i Virgili \\ marialuisa.panisello@urv.cat - inma.pastor@urv.cat \\ marisa.mateu@urv.cat - amaialas@hotmail.com
}

Recibido: 23-02-2010

Aceptado: 16-04-2010

\section{Resumen}

El artículo presenta los resultados de un estudio realizado en la Escuela Universitaria de Enfermería del Campus Terres de l'Ebre de la Universidad Rovira i Virgili (Tarragona), con el objetivo de saber si el conocimiento que existe sobre las diferencias y desigualdades por razón de sexo/género en relación con los problemas de salud, está presente en la formación del alumnado diplomado en enfermería. Dada la actual implantación de nuevos planes de estudios dentro del marco de la convergencia europea de enseñanzas superiores entendemos que es el momento de incidir en la incorporación de la perspectiva de género en el estudio de la salud para adecuar la formación a las necesidades de salud de la comunidad.

Palabras clave: sexo/género, salud-enfermedad, diferencia, desigualdad, estudios enfermería, convergencia europea. 


\begin{abstract}
The article presents the results of a study carried out at the University of Rovira i Virgili Nursing School (Campus Terres de l'Ebre), in order to know whether the existing knowledge about the differences and inequalities related to sex and/or gender related to health problems are present in the Nursing Career formation. Given the current implementation of new curricula within the framework of the European convergence on higher education we understand it is time to influence the incorporation of the gender perspective in health study to adjust the formation to the health needs of the community.
\end{abstract} Key words: sex/gender, health-disease, difference, inequality, nursing curriculum, European convergence.

\title{
1. Introducción
}

Según la Ley de ordenación de las profesiones sanitarias (Ley 44/2003, BOE núm. 280 de 22-XI-2003) corresponde a los diplomados universitarios de enfermería la dirección, la evaluación y la prestación de los cuidados de enfermería orientados a promover, mantener y recuperar la salud, a prevenir enfermedades y discapacidades. Es función de las escuelas universitarias de enfermería formar a los/as estudiantes para adquirir las competencias que los capacitarán para cuidar a los hombres y las mujeres a lo largo de todo el ciclo vital ${ }^{1}$. Por este motivo, es necesario adaptar el currículum de enfermería a la evolución de los conocimientos generados por las investigaciones que se realizan en el campo de la salud y a las necesidades reales que se dan en la dinámica de nuestra sociedad.

La salud es, según consideraciones de la Organización Mundial de la Salud (OMS, 1948), un proceso complejo determinado por la interrelación de factores

\footnotetext{
${ }^{1}$ Guía de Centro. Curso 2006-2007.
} 
biológicos, psicológicos y sociales, y para llevar a cabo los procesos de cuidado de las personas es necesario analizar la interrelación de estos factores; si no es así, estamos sesgando nuestra práctica. A partir de los años noventa del s. XX hay estudios (CAPS, 1991; Wilkinson, 1996; Valls-Llobet, 1998; Marrugat et al., 1998) que muestran que las mujeres y los hombres tienen diferentes maneras de enfermar y de metabolizar los fármacos, así como diferentes estilos de vida, lo que pone en evidencia cómo el género es un determinante en el proceso salud-enfermedad, que produce desigualdades en el acceso a los hospitales, en las listas de espera, en las estrategias terapéuticas, etc. Existen, por tanto, una serie de elementos que intervienen en la salud y en la atención sanitaria que dan como resultado diferencias y desigualdades en la salud y en la atención por razón de sexo/género ${ }^{2}$.

Ante estos estudios, algunos organismos internacionales y gobiernos promueven la introducción de la perspectiva de género para hacer frente a las desigualdades que se derivan de los diferentes roles de las mujeres y los hombres, o de las desiguales relaciones de poder entre ellos y las consecuencias de éstas en sus vidas, salud y bienestar. Así, la OMS elaboró en 2002 un documento para orientar la integración de la perspectiva de género en sus políticas y actuaciones. Igualmente, en la Declaración del Milenio de Naciones Unidas y en otros acuerdos internacionales se reconoce la importancia de la igualdad de derechos entre hombres y mujeres y el derecho de todo el mundo a vivir sin sufrir discriminaciones en ninguna esfera de la vida, incluyendo el acceso a la atención de la salud.

En el ámbito europeo, esta preocupación queda patente en las diversas conclusiones del Consejo sobre la Salud de las Mujeres (2006/C 146/02) (Diario Oficial de la Unión Europea del 22-6-2006). Hay que destacar el compromiso adquirido también por el Acuerdo del Consejo de Ministros de España y publicado en la Orden PRE/525/2005, de 7 de marzo, por la cual, se acuerda la elaboración de un informe

\footnotetext{
${ }^{2}$ Utilizamos el sistema sexo/género porque la salud se relaciona con el género como construcción social y con el sexo diferencias físicas, anatómicas y fisiológicas entre hombre y mujer.
} 
anual sobre salud y género. En Ley orgánica 3/2007 de 22 de marzo para la igualdad efectiva de mujeres y hombres (BOE núm. 71 de 23-3-2007), el artículo 27 hace referencia a la "Integración del principio de igualdad en la política de salud" y destaca que las Administraciones públicas garantizarán igual derecho a la salud de las mujeres y hombres integrando en los objetivos y actuaciones el principio de igualdad de trato, evitando que por las diferencias biológicas o por los estereotipos sociales asociados se produzcan discriminaciones.

El 1r Congreso "Los estudios sobre las mujeres, de género y feministas. Grados y postgrados en el Espacio Europeo de Educación Superior" ${ }^{3}$, que tuvo lugar en Madrid en 2006, reunió profesoras e investigadoras de estudios de las mujeres, de género y feministas de las diversas áreas del conocimiento acordando proponer que en las directrices que el gobierno elabore y remita a las universidades para el diseño de todas las titulaciones de grado se incorporen materias especializadas en estudios de género. Esta propuesta encuentra apoyo, en estos momentos, en la Ley para la igualdad efectiva entre mujeres y hombres, concretamente en el artículo 25: "La igualdad en el ámbito de la educación superior” explicita en el 25.1 "en el ámbito de la educación superior, las administraciones públicas competentes fomentarán la enseñanza y la investigación sobre el significado y el alcance de la igualdad entre mujeres y hombres”. En el punto 2 del mismo artículo se detalla que con tal finalidad, las Administraciones pública promoverán: “a) La inclusión, en los planes de estudio en que proceda, de enseñanzas en materia de igualdad entre mujeres y hombres. b) La creación de postgrados específicos. c) La realización de estudios e investigaciones especializadas en la materia”. Además en el preámbulo la Ley Orgánica de Universidades (LOU) (121/000101, BOE núm. 101-14, 4-IV-2007) dice: "Esta ley no olvida el papel de la universidad como transmisora esencial de valores. El reto de la

\footnotetext{
${ }^{3}$ Organizado por el Ministerio de Trabajo y Asuntos Sociales, la Secretaría General de Políticas de Igualdad, el Instituto de la Mujer, el Ministerio de Educación y Ciencia, y la Unidad de Mujeres y Ciencia.
} 
sociedad actual por lograr una sociedad tolerante e igualitaria, en que se respeten los derechos y libertades fundamentales y de igualdad entre hombres y mujeres"

La URV, como muchas otras universidades, está trabajando en la elaboración de las nuevas titulaciones de grado dando así respuesta a la adaptación al Espacio Europeo de Educación Superior ${ }^{4}$. Es el momento de incidir en el estudio de la salud y los cuidados bajo la perspectiva de género para mejorar y adecuar la formación de los futuros profesionales a las demandas reales de salud de la comunidad para, así, promover un aumento de la calidad de vida de las personas. Como profesoras de los estudios en enfermería de la URV, la pregunta que nos formulamos es: ¿Están suficientemente presentes en la formación de los actuales estudiantes de enfermería el conocimiento que hay en este momento sobre la interrelación sexo/género y la salud?

\section{Antecedentes}

Para entender el conocimiento actual relativo a la salud en relación con el sexo/género, hace falta remontarnos a los antecedentes y la evolución de la situación de las mujeres históricamente y analizar cómo ha influido su posición social en el proceso salud-enfermedad. Dos hechos caracterizan esta situación, por un lado la histórica exclusión de las mujeres de la práctica médica, por otro lado el androcentrismo existente en las ciencias de la salud. Sin embargo, a partir del siglo XX con la aparición de teorías y la articulación social del movimiento feminista, se presentan diversas alternativas al pensamiento androcéntrico. Este hecho permite el desarrollo de estudios que analizan la salud desde la perspectiva de género.

Los movimientos de mujeres desde el s. XIX promueven un pensamiento alternativo al androcentrismo. Los primeros movimientos (también identificados como la primera ola del feminismo) reivindican los derechos de las mujeres; posteriormente,

\footnotetext{
${ }^{4}$ Adaptación para el curso 2009-2010.
} 
la segunda ola del feminismo hará posible generar y producir teorías explicativas de la desigualdad entre hombres y mujeres. Las feministas de finales del siglo XIX y primera mitad del siglo XX centraron su lucha en la reivindicación del derecho al voto y la igualdad jurídica y política de las mujeres, pero no fue hasta el 26 de agosto de 1920 que se puede leer en la constitución norteamericana "El derecho de los ciudadanos de los Estados Unidos al voto no puede ser negado o limitado por los Estados Unidos por ningún motivo, debido a la diferencia de sexo" (enmienda núm. 19 de la Constitución norteamericana). En España será en 1931, con la II República, cuando las mujeres puedan ejercer su derecho al voto por primera vez.

Será con el desarrollo de las teorías feministas del siglo XX cuando los estudios realizados en relación a la atención sanitaria prestada a las mujeres, el análisis de los procesos de salud-enfermedad y los movimientos de mujeres y salud, interesadas en el control del propio cuerpo, han permitido la desconstrucción de los paradigmas asociados al patriarcado. En este contexto se plantean alternativas al pensamiento androcéntrico, y se generan nuevas hipótesis que ponen en evidencia la invisibilidad de las mujeres. Sin embargo, sigue siendo necesario profundizar en estas explicaciones y alternativas a los procesos de salud y enfermedad en los cuales no se puede obviar la influencia del género como elemento constitutivo y generador de desigualdades. El Colectivo de Mujeres de Boston (Boston Women's Health Collective) representa una de las experiencias más emblemáticas. El libro Nuestros cuerpos, nuestras vidas, fue el resultado de un pequeño grupo de discusión sobre "las mujeres y sus cuerpos" que formó parte de una conferencia pronunciada en Boston en el año 1969. El libro trata sobre el cuerpo de las mujeres, la maternidad, las relaciones sexuales, la salud y las enfermedades más frecuentes. El último capítulo aborda el tema de la mujer y la salud pública $\mathrm{y}$, desde su experiencia, reflejan su pensamiento crítico con la conceptualización de las mujeres y de sus cuerpos por parte de la sanidad y de los y las profesionales sanitarios/arias, proponiendo información y estrategias para resolver estos problemas. (Colectivo del Libro de Salud de las Mujeres de Boston, 1982). 
Hohmann (1989) ${ }^{5}$ hizo una primera propuesta de las áreas en las cuales haría falta realizar formación sobre la relación mujer y salud:

- Enfermedades cardíacas (enfermedad de arterias coronarias, dislipémias).

- Enfermedades endocrinas (tiroides, diabetes, hirsutismo).

- Enfermedades infecciosas (SIDA, enfermedades transmitidas sexualmente).

- Enfermedades de las vías urinarias (incontinencia, infección de las vías urinarias).

- Enfermedades neurológicas (migraña, síndrome de fatiga crónica)

- Enfermedades musculoesqueléticas (osteoporosis, lupus, fibromialgia, síndrome del canal carpiano).

- $\quad$ Problemas en las mamas (benignas, malignas, galactorrea)

- Salud reproductiva (contracepción, salud pregestacional y postgestacional, embarazo de alto riesgo).

- Menopausia.

- $\quad$ Sexualidad.

- Enfermedades que afectan a la vagina y a la cérvix (vaginitis, displasia cervical).

- Anomalías pelvianas (enfermedades inflamatorias de la pelvis, fibromas, cáncer uterino).

- Anomalías de la menstruación (amenorrea, metrorragias, endometriosis, síndrome premenstrual).

- Trastornos psiquiátricos (depresión, ansiedad, trastornos de la conducta alimentaria, anorexia y bulimia).

- Violencia doméstica.

- Adicciones a drogas y fármacos.

${ }^{5}$ Referenciado por Valls. Investigación y planes de docencia libres de sesgos de género en salud. IV Congreso Internacional de Universidades promotoras de Salud. Pamplona 2009 
- Mujeres en la medicina (problemáticas profesional y personal).

Actualmente haría falta revisarlas y profundizar en la relación de la satisfacción de las necesidades, para dar una atención integral con una visión de sexo/género. En nuestra propuesta añadiríamos otros elementos que consideramos que requieren una revisión desde la perspectiva de género:

- El estudio de los patrones de vida, puesto que tienen una clara distribución por género (conductos de riesgo, falta de equidad en la distribución de los tiempos de trabajo productivo y reproductivo, de ocio, de descanso,...).

- El estudio de las segregaciones horizontales y verticales en relación con el trabajo productivo que implican que mujeres y hombres estén expuestos en diferentes riesgos físicos, psicosociales y obtengan diferentes salarios.

- El análisis del trabajo reproductivo, puesto que la actual división del reparto de los tiempos supone una sobrecarga física, y emocional y un impacto en la salud de las mujeres.

- El estudio de las diferencias y desigualdades en cuanto a la accesibilidad, el esfuerzo diagnóstico y terapéutico.

- Mujeres y enfermería (historia de la profesión, como influye el género en la relación terapéutica, profesión y género....).

La Food and Drug Administration (FDA), agencia americana que prueba la utilización de nuevos tratamientos, publicó una guía en 1977, que excluía explícitamente de los ensayos clínicos por considerarlas dentro de los grupos vulnerables por la investigación, a las mujeres en edad fértil como prevención de un riesgo potencial de daño fetal. Haciendo una valoración sobre los resultados de esta decisión, en el desarrollo del conocimiento sobre la salud de las mujeres, este hecho ha podido perjudicar más que proteger. El año 1993 la FDA, eliminó esta prohibición e instó a incluir mujeres en todos los ensayos. Más tarde en 1994, el Instituto Nacional de 
Salud de los Estados Unidos publicó otra guía donde se recomienda la inclusión de mujeres y minorías como sujetos de investigación clínica (Artazcoz, 2005).

Un paso más en la consolidación de los conocimientos científicos en la relación del sexo/género y la salud fueron las redes internacionales que durante el año 1990 se reunieron en varios lugares del mundo: Barcelona, con el Primer Simposio Internacional sobre Mujer y Calidad de vida, organizado por el Centro de Análisis de Programas Sanitarios (CAPS), Women's Health Meeting en Boston. (Cuaderno CAPS, 1991). Otro elemento a destacar es la inclusión del ítem Women's Health (salud de las mujeres) en el Index Medicus en el año 1991. En el mismo año 1991, Bernardine Healy puso en evidencia la mala praxis de los servicios de cardiología americanos por no realizar las mismas técnicas diagnósticas ni las mismas exploraciones a mujeres que a hombres. Aquel análisis mostró que se practicaban muchas más coronariografias a los hombres que a las mujeres y que las mujeres estaban excluidas sistemáticamente de los ensayos clínicos. La Conferencia anual de la British Psychological Society del año 1992 también trató sobre el tema de "Mujer y salud: un enfoque feminista" (Wilkinson, Kitzinger, 1994).

Todos estos movimientos de mujeres destacan por ser el inicio y el referente en la construcción del conocimiento de como influye el género en la salud y los problemas de salud. Marchitaba Angell, en el editorial del New England Journal of Medicine (julio de 1993), analiza la discriminación de las mujeres en la investigación médica y señala que la discriminación se produce en la medida que se constatan tres hechos ${ }^{6}$ :

1. Las enfermedades que afectan más a las mujeres han sido menos estudiadas.

2. Es menos probable que las mujeres estén incluidas como participantes en ensayos clínicos.

3. Es improbable que consigan ser directoras de investigaciones para llevar a término ensayos clínicos.

\footnotetext{
${ }^{6}$ Referenciado por Valls (2001: 181).
} 
La formulación y el desarrollo de la teoría de los géneros en la década de los setenta, abrió un gran campo de estudio de como influye el sexo/género en la salud evidenciando las desigualdades existentes. En las descripciones etnográficas y la observación de otras culturas, en que se descubrieron sociedades con más de dos géneros se demuestra que puede haber diversidad de contenidos en aquello que se considera masculino y femenino en las diferentes culturas y épocas. Este descubrimiento contribuyó a deconstruir el supuesto carácter natural de las identidades masculinas y femeninas ${ }^{7}$.

Este sistema conceptual (sexo/género) pone en entredicho la supuesta base biológica de los comportamientos masculinos y femeninos y afirma que el género es una construcción social, es decir, que el que se entiende que es un hombre o una mujer no son un conjunto de datos anatómicos, sino construcciones sociales y culturales con un apoyo biológico ambiguo e inestable (Rivera, 2003). En concreto, Rubin (1975) define el sistema sexo/género como el sistema de relaciones sociales que transforma la sexualidad biológica, que no se debe confundir con el sexo, en un producto de la actividad humana: en cuanto a la división sexual del trabajo y la orientación heterosexual del erotismo. Para Butler (1990), en el proceso de constitución de la subjetividad intervienen relaciones de poder que nos constituyen como el que somos: mujeres $\mathrm{u}$ hombres. Nos hace ser mujeres $\mathrm{u}$ hombres el someternos a las reglamentaciones del género: la mujer es hacer de mujer y hombre es hacer de hombre ${ }^{8}$.

Tal y como nos recuerda Izquierdo (2003) el género es un concepto que logra una pluralidad de dimensiones: la física (que denominamos sexo), la psíquica, la social, la política, la cultural, o la económica, por denominar las principales, pero destacan, llegando a condicionar los restantes ámbitos: la manera como las personas se relacionan

\footnotetext{
${ }^{7}$ Los antecedentes a la teoría de los géneros hace falta buscarlos en los estudios antropológicos de Margaret Mead y en El segundo sexo de Simone de Beauvoir con la afirmación: "La mujer no nace, sino que se hace".

${ }^{8}$ Referenciado por Izquierdo (2003: 27).
} 
en la producción, la orientación de su deseo y la pauta predominante que guía las acciones. El que denominamos mujer y hombre no son sujetos sometidos a relaciones desiguales, sino efectos del poder, materializaciones de la desigualdad social. Dentro de la dimensión social del género:

"Que los hombres y las mujeres ocupan diferentes posiciones sociales no ha pasado de manera espontánea, sino que es el resultado del ejercicio del control social. Forman parte del control social, garantizando que las personas se ajustan a unas ciertas exigencias sociales: el sistema educativo, la familia, la organización del trabajo, el tratamiento de las diversidades culturales, los medios de comunicación, etc.” (Izquierdo, 2003:31).

La interiorización del género a través de los componentes afectivos y cognitivos se produce en la primera niñez e implica la asunción de pertenencia a un sexo por diferencia del otro, el género tiene una vertiente colectiva que presupone la adaptación de los sujetos a las expectativas de la cultura donde se nace y crece, y una vertiente individual que es cómo y en qué medida vive cada cual su género (Sau, 1993). Para Scott (1988), las teorías sociológica y sociobiológica de género son las más utilizadas para explicar las diferencias y desigualdades en la asignación de roles desarrollados por hombres y mujeres en la sociedad. La teoría sociobiológica se fundamenta en el hecho que las diferencias entre hombres y mujeres son de carácter biológico y no social. Por su parte, la teoría sociológica no descarta la importancia de las diferencias biológicas, pero considera que los principales factores que condicionan las desigualdades de género son de origen social y político (Rohlfs, et al., 2000).

Así, los ejes básicos de la conceptualización del género: socialización, roles y normas que son desiguales para hombres y mujeres, inciden tanto en la estructura de oportunidades como en la división social del trabajo. De este modo se manifiestan desigualdades sociales con respecto a la perspectiva de vida que incluyen, por un lado, la educación, la ocupación y los sueldos, y por la otra, la organización del trabajo 
reproductivo. Las ocupaciones están claramente matizadas por los roles sociales que sistemáticamente dependen del género (Rohlfs, Borell y Fonseca, 2000).

\section{Estado actual del tema}

En este apartado resumimos los conocimientos sobre desigualdades y diferencias en la salud en relación con el sexo/género y mostramos los indicadores de mortalidad, esperanza de vida, autopercepción de la salud y morbilidad, así como los elementos que intervienen en la prevención de los problemas de salud y en la promoción de la salud en relación con el género.

\subsection{Elementos propios de género relacionados con la salud}

Para entender las desigualdades en salud según el género, principalmente las relacionadas con el estado de salud percibido (Arber, 1991) ${ }^{9}$, señala la importancia de factores como la clase social, la ocupación, la educación, los patrones de consumo y la propiedad de bienes. Las políticas sociales son uno de los mecanismos distribuidores de la riqueza de un estado. Al hablar de desigualdades hay que recordar que la forma y amplitud del estado de bienestar no sólo influye sobre las desigualdades de clase social, sino también sobre las de género, puesto que algunas intervenciones afectan especialmente a las condiciones de vida de las mujeres como es el caso de los servicios a las familias. En España, el escaso nivel de desarrollo de los servicios a las familias y la sobrecarga de responsabilidades, y en particular a las mujeres, es elevado. La ausencia de ayuda a la familia crea una sobrecarga a la mujer española, que cuida a los niños, los jóvenes, los maridos o compañeros, las personas mayores y discapacitadas y un $42 \%$ además tiene ocupación remunerada, cosa que significa una enorme sobrecarga

\footnotetext{
${ }^{9}$ Referenciado por Rohlfs I, et al. 2000.
} 
(Navarro et al., 2004). Según la Encuesta Nacional de Salud del 2006 (ENS, 06) el $50,8 \%$ de los hombres y el 85,9\% de las mujeres que conviven con menores de 15 años se encargan de su cuidado. Los hombres dedican, de media, 40 horas semanales, y las mujeres, 65. El 32,4\% de los hombres y el 58,5\% de las mujeres que conviven con mayores de 74 años se encargan de su cuidado. La dedicación media, en horas semanales, es de 66 en el caso del hombre y 68 en el de las mujeres. El 33,2\% de los hombres y el $64,3 \%$ de las mujeres que conviven con personas con discapacidad se encargan de su cuidado. Mientras que los hombres dedican, de media, 65 horas semanales, las mujeres, 71. El 35,9\% de los hombres y el 81,5\% de las mujeres de 16 y más años realizan tareas del hogar, tales como limpiar, cocinar o planchar. Los hombres y las mujeres que realizan tareas del hogar dedican, de media, 15 horas semanales en el caso de los hombres y 28 en el de las mujeres.

Estos datos nos recuerdan la importancia de analizar cómo el género se asocia con las circunstancias socioeconómicas individuales y familiares, de forma que a medida que empeoran las condiciones del entorno, los indicadores de salud son más negativos. Por esto, es importante estudiar cómo las condiciones del entorno social, de los trabajos (productivo y reproductivo) y de los hábitos de vida influyen sobre la salud de los individuos en una sociedad profundamente desigual en función del género (Rohlfs, et al., 2000). Según estas autoras, las variables que hay que tener en cuenta para diseñar las encuestas de salud y que posibilitarían el análisis desde la perspectiva de género son las que se citan a continuación en el Cuadro 1: 
Cuadro 1: Variables que posibilitan el análisis desde una perspectiva de género

1. Sociodemográficas y de trabajo:

- Estado civil de hecho

- Edad

- Clase social

- Nivel de estudios

- Situación laboral

- Trabajo productivo (remunerado)

- Ocupación o puesto de trabajo.

- Tipo de contrato.

- Tipo de jornada laboral.

- Horas de trabajo a la semana.

- Trabajo reproductivo (no remunerado)

○ Estructura familiar (convivencia): edad, sexo, parentesco de las personas que conviven.

○ Trabajo doméstico

- Realización del trabajo doméstico: hacer la compra, cocinar, limpiar, planchar...

- Distribución del trabajo doméstico

- Cuidado de las personas dependientes (niños, personas mayores, discapacitados/as)

- Distribución de los cuidados.

- Disponibilidad de ayuda externa (familiar o contratada) parar realizar las tareas domésticas y de cuidados.

○ Horas dedicadas al trabajo reproductivo.

2. Morbilidad, salud percibida, calidad de vida y salud mental. Trastornos crónicos.

3. Apoyo social (presencia y tipo de red social). Apoyo social funcional (afectivo y confidencial)

Fuente: Rohlfs, et al., (2000:149)

\subsection{Indicadores de la situación de salud según sexo/género}

\subsubsection{Esperanza de vida, mortalidad, autopercepción de la salud}

Según el Instituto Nacional de Estadística (2005), la esperanza de vida al nacer de las mujeres es de 83,4 años y la de los hombres 76,9, aunque la esperanza de vida en buena salud es de 53,9 años para las mujeres y 56,3 años para los hombres. Respecto al mantenimiento de las capacidades para actividades de la vida diaria sin necesidad de ayuda, para la población de 65 y más años, el 65,6\% declara que es capaz de realizar las 
actividades relacionadas con el cuidado personal (74\% de los hombres y 59\% de las mujeres). Las mujeres han de afrontar, en mayor proporción que los hombres, el trauma de la viudedad y los pocos recursos económicos, situación que en ocasiones se prolonga durante décadas. Esto comporta un estado de soledad que unido a una salud precaria degrada la calidad de vida. Con la edad se incrementa la pobreza, la soledad y las discapacidades (Mateu, Panisello, Lasaga y Bonfill, 2007). La tasa de mortalidad para ambos sexos es de $8,4 \%$, siendo $7,9 \%$ para las mujeres y $8,9 \%$ para los hombres. La primera causa de muerte en los hombres es la isquemia cardíaca, y en las mujeres la enfermedad cerebrovascular. De los tumores, el que causa más mortalidad en los hombres es el de pulmón, y en las mujeres, el de mama. Con respecto a la percepción de la salud, según la Encuesta Nacional de Salud (ENS, 2006), declaran tener un estado de salud bueno o muy bueno el 75,1\% de los hombres y el $65 \%$ de las mujeres. La percepción de la salud empeora a medida que aumenta la edad y también en las clases sociales más desfavorecidas.

\subsubsection{Morbilidad}

Estudios realizados por Berstein (1990) demostraban que en la década de los ochenta las quejas por dolor y malestar en las mujeres se consideraban predominantemente psicosomáticas y se trataban con ansiolíticos y sedantes ${ }^{10}$. Son muchas las enfermedades que pueden causar dolor muscular y articular y hay que tenerlas en cuenta (Valls-Llobet, 2006), en el libro Mujeres invisibles, nos habla de la morbilidad femenina diferencial como el conjunto de enfermedades, motivos de consulta o factores de riesgo que merecen una atención específica hacia las mujeres, porque son problemas exclusivamente de las mujeres o se presentan más frecuentemente.

En la Encuesta Nacional de Salud (ENS 2006) se ha explorado la salud mental de la población, mediante el cuestionario GHQ-12, el 21,3\% de la población de 16 años o más $(15,6 \%$ de los hombres y $26,8 \%$ de las mujeres) presenta riesgo de mala salud

\footnotetext{
${ }^{10}$ Referenciado por Valls (2006: 201).
} 
mental, siendo este riesgo mayor a medida que aumenta la edad. Así, en el grupo de edad de 75 y más años, lo presentan el 25,0\% de los hombres y el 39,5\% de las mujeres.

Hay que señalar que el $76 \%$ de las mujeres cuidadoras de enfermos de demencia presentaban sensaciones de depresión (Panisello, Lasaga, Mateu, 2000). El incremento de la morbilidad psiquiátrica por edades en mujeres casadas en comparación con las solteras es un hecho frecuentemente observado y remarca la importancia en la formación de los profesionales de psiquiatría en las diferencias de género (Montero, et al., 2004).

El Plan de Salud de Cataluña (2002-2005) señala que la violencia doméstica ${ }^{11}$ es un problema que repercute de forma determinante en la salud y la calidad de vida de las personas que la sufren. La magnitud de este problema no se conoce con exactitud. La OMS define la violencia de género como el uso intencional de la fuerza física o poder, real o mediante amenaza, contra otra persona y basado en el género, que puede resultar lesionada con daño o padecimiento físico, sexual o psicológico, o coacciones, o privada de libertad y que, en el peor de los casos, puede provocar la muerte de las víctimas. Según el informe de la Asamblea Parlamentaria del Consejo de Europa de septiembre de 2002 en los países de la Unión Europea la violencia de género es la principal causa de muerte o incapacidad entre las mujeres europeas de 16 a 44 años. La violencia contra las mujeres en la pareja tiene su origen en las relaciones de poder desiguales entre hombres y mujeres. Según las Naciones Unidas, hasta 1991 solamente 22 países del mundo industrializado habían concedido a las mujeres iguales derechos que a los hombres en cuestión de matrimonio, divorcio y propiedad familiar (RuizPerez, Blanco-Prieto y Vives-Cases, 2004).

La violencia contra las mujeres es una violencia estructural porque tiene su origen y se fundamenta en las normas y valores sociales y culturales que determina el

\footnotetext{
${ }^{11}$ Termino utilizado en aquella época. El 8 de Marzo de 1998 se presenta, en el estado español, el I Plan contra la Violencia Doméstica y en el año 2001 se aprueba el II Plan contra la Violencia Doméstica (2001-2004). Con la aprobación en Diciembre del 2004 de la Ley integral Contra la Violencia de Género, cambia el marco interpretativo y supone finalmente la satisfacción de una demanda de los colectivos feministas. Bustelo (2006: 85).
} 
orden social patriarcal establecido desde hace miles de años, no ha sido determinado por la naturaleza humana (Valls-Llobet, 2006). La mejor manera de conceptualizar la violencia contra las mujeres es considerarla un factor de riesgo para la salud, puesto que sus consecuencias implican una amplia gama de efectos en la salud de las mujeres (Garcia-Moreno, 2000).

\subsubsection{Estilos de vida}

La adopción de estilos de vida diferentes por mujeres y hombres está influida por las normas y roles establecidos socialmente y pueden dar lugar a desigualdades en salud (Laceras ML, Pires m, Rodríguez MM., 2004). Según la Encuesta Nacional de Salud (ENS 2006), en relación a los hábitos de vida que están relacionados con la salud, el $26,4 \%$ de la población de 16 y más años afirma que fuma a diario, el 3,1\% es fumador/a ocasional, el 20,5\% se declara exfumador/a y el 50,0\% dice que nunca ha fumado. Por sexo, el porcentaje de fumadores es del 31,6\% de los hombres y del 21,5\% de las mujeres. El porcentaje de personas que presentan un consumo de bebidas alcohólicas en cantidades consideradas de riesgo para la salud se sitúa en el 6,8\% de los hombres y en el $2,7 \%$ de las mujeres.

La realización de actividad física es uno de los principales determinantes de la salud en los países desarrollados y, en relación a esto, se constata que las mujeres realizan menos actividades físicas que los hombres en su tiempo libre.

\subsubsection{Actividades preventivas}

Haría falta estudiar las influencias de sexo/género en la historia de los problemas de salud para poder después llevar a cabo actividades preventivas en relación con los niveles de prevención.

\subsubsection{Promoción de la salud}

La estrategia se centra en la reducción de las desigualdades en salud arraigadas en la falta de equidad social, el punto clave está en la carencia de poder y 
en la desigualdad de oportunidades históricamente configuradas (Mazarrasa, 2001). No puede haber una buena promoción de la salud con perspectiva de género sin una mejor organización de los servicios sociales y sin combatir el estrés psicosocial que se genera por las condiciones diferentes de vida y trabajo de las mujeres, factor esencial para mejorar su salud. Por esto las intervenciones dependerán de las leyes, de los recursos sociales y de la necesaria coordinación sanitaria. Por otra parte, la organización sanitaria y todo el sistema que en principio debería ser un factor más de promoción de la salud se convierte a veces en un sistema negativo para las mujeres porque medicaliza muchos de los procesos fisiológicos como son el embarazo, el parto y la menopausia, y en cambio no atiende correctamente los problemas que tienen una morbilidad diferencial porque no han sido estudiados desde esta perspectiva (Valls-Llobet, 2006).

\subsubsection{Utilización del sistema sanitario}

En cuanto a la utilización del sistema sanitario, los hombres presentan más ingresos hospitalarios para la mayoría de grupos diagnósticos, siendo las enfermedades del aparato digestivo la primera causa de hospitalización, seguidas del circulatorio y respiratorio. En las mujeres la primera causa motivo de ingreso es el parto, el puerperio y las complicaciones del embarazo, seguida por las enfermedades del aparato circulatorio y digestivo. Sin embargo, en un estudio realizado de las solicitudes de primera visita para las consultas externas del área médica de un hospital, el 53,8\% eran mujeres frente al 46,2\% que eran hombres (Rosa-Jiménez F, Montijano AM, Herraiz C, Zambrana JL, 2005).

\subsubsection{Política sanitaria y práctica profesional en relación con el género}

La atención sanitaria contribuye a las desigualdades en salud asociadas al género. Ruiz-Cantero (2004) define el sesgo de género cómo: “El planteamiento erróneo de igualdad o de diferencia entre hombres y mujeres, en cuanto a su naturaleza, sus comportamientos y/o sus razonamientos, que puede generar una conducta desigual en los servicios sanitarios incluyendo la investigación, y que es discriminatorio de un sexo 
respecto del otro" (Ruiz-Cantero y Verdú-Delgado, 2004: 119). La Ley general de sanidad de 1986 estableció los principios de las políticas sanitarias de las comunidades autónomas a través de los planes de salud. El estudio realizado por Peiró et al. (2004) muestra importantes diferencias con respecto a la sensibilidad de género (el grado en que los planes de salud tienen en cuenta la existencia del género como categoría de relación y desarrollan acciones para aminorar las desigualdades de esta consecuencia) y también que la inclusión de objetivos generales de equidad de género no suelen acompañarse del desarrollo de acciones específicas operativas.

La información de los problemas de salud se suele presentar desglosada por sexos (el tabaquismo, la enfermedad oncológica y los accidentes de tráfico son los problemas de salud que más presentan información por sexo) pero la sensibilidad de género disminuye cuando se trata de formular acciones y objetivos para el desarrollo de los planes (Peiró, et al., 2004).

\section{Objetivos, hipótesis y metodología}

El objetivo general de este artículo es identificar si las diferencias y desigualdades por razón de sexo/género en relación con los problemas de salud, están presentes en la formación de los/las diplomados/das de enfermería de la Escuela Universitaria de Enfermería del Campus Terres de l'Ebre (EUETE) de la Universidad Rovira i Virgili (URV). Este objetivo se complementa con otros más específicos que son, en primer lugar, analizar lingüísticamente la presencia del sexo/género en los programas de formación de los estudios de enfermería publicados en las guías de los estudios de la diplomatura, en segundo lugar, identificar si están presentes, en los programas de las diferentes asignaturas curriculares de los estudios de la diplomatura de enfermería, los conocimientos sobre las desigualdades y las diferencias en salud por razón de sexo/género y, finalmente, conocer si el alumnado de tercer curso tiene los 
conocimientos sobre las desigualdades y diferencias por razón de sexo/género en cuanto a los problemas de salud.

Nuestra hipótesis general de partida es que el conocimiento que tenemos actualmente sobre las diferencias y las desigualdades por razón de sexo/género en relación con el proceso salud-enfermedad no está presente en la formación actual de los diplomados/as de enfermería. El proceso de formación comienza con el diseño de los programas que forman el currículum de un estudio y que se implantará a lo largo de toda la carrera, por eso esta hipótesis general la hemos subdividido en tres subhipótesis relacionadas, respectivamente, con el lenguaje utilizado, con los contenidos del plan de estudios y con el conocimiento que al final tiene el alumnado. Las subhipótesis que hemos formulado para aceptar o rechazar la hipótesis general son: "En los programas de formación del diplomado/a de enfermería, la mayoría de asignaturas utilizan, alguna vez o más, el masculino para referirse a los dos géneros", "En los programas de formación del diplomado/a de enfermería no están presentes los conocimientos existentes sobre las desigualdades y diferencias por razón de sexo/género en relación con el proceso saludenfermedad" y que "El alumnado de tercer curso no es conocedor de las desigualdades y diferencias por razón de sexo/género existentes actualmente en relación con el proceso salud-enfermedad".

El estudio se llevó a cabo en la Escuela Universitaria de Enfermería (EUE) de Tortosa, de la URV ${ }^{12}$. En cuanto a la metodología utilizada se planteó con un enfoque cualitativo y eso implica la utilización de técnicas cualitativas de análisis documental (los programas) y entrevistas semiestructuradas por grupos o redacción colectiva. Para dar respuesta a los objetivos planteados y a las hipótesis formuladas se decidió que nuestra población de estudio estuviera formada por los programas de las diferentes asignaturas de los tres cursos de los estudios de la diplomatura de enfermería de la EUE

\footnotetext{
${ }^{12}$ La EUE de la URV está formada por dos centros, el centro situado en la ciudad de Tarragona y el de la ciudad de Tortosa, que forma parte del Campus Terres de l'Ebre (TE), en el cual se imparte la Diplomatura de Enfermería con 219 alumnos matriculados en el curso académico (2006-07).
} 
del Campus Terres de l'Ebre de la URV y el alumnado de tercer curso de la diplomatura de enfermería de la referida Escuela.

Las variables estudiadas son:

- Las expresiones lingüísticas en relación al género.

- La presencia en los programas de los conocimientos existentes en relación a las desigualdades y diferencias en salud por razón de sexo/género.

- El grado de conocimiento de las diferencias y desigualdades por razón de sexo/género que tienen los alumnos de tercer curso.

Los instrumentos utilizados para la recogida de información para analizar las variables son unas fichas diseñadas para el análisis lingüístico, otras diseñadas para el análisis de contenidos de los programas y una entrevista semiestructurada por grupos o redacción colectiva del alumnado de tercer curso.

Para analizar las variables hemos utilizado las técnicas siguientes:

1) Análisis lingüístico de los programas

Como ya se ha apuntado, una de las variables a estudiar es el uso del género lingüístico en los programas. La utilización del lenguaje en masculino como consecuencia del androcentrismo, conlleva una discriminación implícita y continúa presente en la redacción de documentos, manifestándose sobre todo con la presencia continua del masculino y la ocultación sistemática del género femenino. De acuerdo con el objetivo del estudio, un primer paso ha sido analizar lingüísticamente los programas incluidos en la Guía del centro de la EUE, de los tres cursos de la diplomatura de enfermería.

2) Análisis de los contenidos de los programas

Tanto en la introducción como en la primera parte de este artículo se han identificado los conocimientos actuales en relación con las diferencias y desigualdades en salud según el sexo/género. La pregunta que nos hacemos es si estos conocimientos están presentes en los programas de formación de la diplomatura de enfermería. Siendo conscientes de que los programas a veces no 
reflejan toda la formación que se desarrolla en un aula, también hemos recogido información relativa al alumnado de tercer curso, para obtener información sobre el grado de conocimiento que tienen sobre el tema que nos ocupa.

3) Entrevista semiestructurada de redacción colectiva al alumnado de tercer curso

La tercera técnica empleada en este trabajo de búsqueda ha sido la entrevista semiestructurada con el objetivo de recoger información sobre los conocimientos que el alumnado tiene de la relación sexo/género y salud y de las desigualdades existentes en salud por razón de género. Se ha elegido el alumnado de tercer curso, porque están al final de los estudios y porque ya han dado todas las asignaturas teóricas de la diplomatura y están finalizando su periodo de prácticas. Para medir el grado de conocimiento en relación con las diferencias y desigualdades por razón de sexo/género que tienen los alumnos de tercer curso de la diplomatura de enfermería y poder aceptar o rechazar la hipótesis a estudiar hemos diseñado un guión de entrevista. La entrevista semiestructurada consta de cinco preguntas abiertas, para que el alumnado pueda contestar todo lo que considere oportuno y no limitar las respuestas. En el Cuadro 2 se presentan las preguntas realizadas.

\section{Cuadro 2 - Entrevista semiestructurada.}

EDAD:

SEXO:

Estamos realizando un estudio sobre el grado de conocimiento que tienen los estudiantes de enfermería sobre la relación sexo / género y la salud.

1. ¿Qué diferencias conoces en cuanto al proceso salud-enfermedad entre hombres y mujeres?

2. ¿Como explicarías esta diferencia?

3. ¿Más allá de las diferencias en el proceso salud-enfermedad, crees que hay diferencias y desigualdades entre hombres y mujeres en cuanto al diagnóstico, tratamiento, trato, listas de espera, etc. dentro del sistema sanitario?

4. ¿En qué asignaturas has estudiado o comentado estas diferencias?

5. ¿Has leído bibliografía específica sobre esta cuestión? ¿Puedes referenciar algún autor/a?

Fuente: elaboración propia 


\section{Discusión de los resultados}

De las 30 asignaturas que forman el curriculum de los estudios de la diplomatura, $7(23,4 \%)$ utilizan siempre genéricos en referirse a los dos géneros; las 23 asignaturas restantes (76,6\%), alguna vez o más utilizan el masculino para referirse a los dos géneros. Con estos resultados podemos aceptar nuestra hipótesis de partida: En los programas de formación del diplomado/da de enfermería, la mayoría de asignaturas utilizan, alguna vez o más, el masculino para referirse a los dos géneros.

En relación a los contenidos de los programas en 5 asignaturas no es relevante que no aparezca alguna mención al sexo/género por el contenido de la materia (biestadística), en las 25 restantes, tan sólo en 2 (8\%), se hace mención al sexo/género de manera explicita. Constatamos así que, el 92\% de las programas de las asignaturas del currículum de la diplomatura de enfermería no hace mención a la relación sexo/género y el proceso salud enfermedad de una manera explicita. Con estos resultados también podemos aceptar nuestra hipótesis de partida en la que apuntábamos que: En los programas de formación del diplomado/a de enfermería no están presentes los conocimientos existentes sobre las desigualdades y diferencias por razón de sexo/género en relación con el proceso salud-enfermedad.

En relación a los resultados de la entrevista ${ }^{13}$, para facilitar el análisis, hemos clasificado las respuestas a la primera pregunta sobre el conocimiento de las diferencias entre hombre y mujeres en cuanto al proceso salud-enfermedad, en cuatro grupos. Por un lado se ha considerado aquellas respuestas que constatan que sí existen diferencias entre hombres y mujeres en algunos problemas de salud; así el 60,2\% de las respuestas dadas señalan que hay problemas de salud en los que la frecuencia es diferente en hombres y mujeres, sobre todo los tipos de cánceres. En segundo lugar se han considerado las respuestas que describen o manifiestan esta diferencia en relación al proceso salud-enfermedad; así hay un $31,8 \%$ de respuestas que nos dicen que las

\footnotetext{
${ }^{13}$ De los 73 alumnos/as matriculados en tercer curso, contestaron 39 (53,4\%). La edad media es de 21,5 años con un rango de 20-27. Siendo 35 mujeres y 4 hombres.
} 
diferencias se manifiestan en que las mujeres tienen más esperanza de vida y más morbilidad. El tercer y cuarto tipo de respuestas son minoritarias. En concreto, el tercer grupo de respuestas son las que contestan que hay otras variables que inciden en el proceso salud-enfermedad: específicamente un 5,6\% de las respuestas señalan que hay otras variables como la edad, estilos de vida y el carácter de cada uno/a que inciden en la salud-enfermedad. Finalmente, un cuarto grupo de respuestas son la que contestan que las diferencias dependen de elementos relacionados con el rol de género: en concreto solo un $2,2 \%$ de las respuestas dicen que las diferencias dependen de elementos relacionados con los roles de género.

Ante la pregunta “¿Cómo explicarías esta diferencia?” el análisis hecho nos permite decir que el 36,9 \% de las respuestas atribuyen esta diferencia a la anatomofisiología de cada sexo. Otro $17.8 \%$ de las respuestas explican las diferencias en base a los estilos de vida. El 20,5\%, atribuyen la razón de esta diferencia a factores relacionados con el rol y un $24,4 \%$ de las respuestas atribuye la diferencia a elementos estructurales de la sociedad. Cabe señalar que un 0,2 no contesta y el 0,2 señalan que no hay diferencias. En relación a la pregunta sobre si encuentran que hay diferencias y desigualdades entre hombres y mujeres en cuanto al diagnóstico, tratamiento, trato, listas de espera..., dentro del sistema sanitario, las respuestas obtenidas se pueden agrupar en 7 categorías de análisis. Los resultados se muestran en la tabla 1.

Tabla 1

\begin{tabular}{|l|c|c|}
\hline Respuestas & Frecuencia absoluta & Frecuencia relativa (\%) \\
\hline No hay diferencia & 29 & $74,3 \%$ \\
\hline No tendría que haber diferencias & 1 & $2,5 \%$ \\
\hline Pueden haber diferencias & 3 & $7,6 \%$ \\
\hline Sí que hay diferencias & 3 & $7,6 \%$ \\
\hline Hay bastante igualdad & 1 & $2,5 \%$ \\
\hline NS (no lo sabe) & 1 & $2,5 \%$ \\
\hline No hay diferencias, pero si desigualdades & 1 & $2,5 \%$ \\
\hline Total & 39 & 100 \\
\hline
\end{tabular}

Fuente: Elaboración propia 
Cabe señalar que el 74,3\% responden que no hay diferencias en relación al diagnóstico, tratamiento, trato, listas de espera..., dentro del sistema sanitario. A este respecto hay que recordar que hay estudios que ponen en evidencia que sí existen estas diferencias (Ruiz-Cantero, 2004; Valls-Llobet, 2006; Peiró, 2004).

Finalmente se quiso averiguar qué asignaturas están facilitando este conocimiento y se preguntó específicamente: ¿En qué asignaturas habéis estudiado o comentado estas diferencias? La gran mayoría del alumnado contestó que en más de una asignatura. Las respuestas se muestran en la tabla 2.

Tabla 2

\begin{tabular}{|l|c|c|}
\hline Asignaturas & Frecuencia absoluta & Frecuencia relativa (\%) \\
\hline Estructura y función cuerpo humano & 4 & $5,0 \%$ \\
\hline Enfermería Medicoquirúrgica & 14 & $17,7 \%$ \\
\hline Enfermería Comunitaria & 33 & $41,7 \%$ \\
\hline Enfermería Geriatríca & 2 & $2,5 \%$ \\
\hline Enfermería Maternoinfantil & 7 & $8,8 \%$ \\
\hline Fundamentos de enfermería & 2 & $2,5 \%$ \\
\hline Enfermería Psicosocial & 6 & $7,5 \%$ \\
\hline Enfermería Psiquiatría & 2 & $2,5 \%$ \\
\hline Evolución sexualidad & 1 & $1,2 \%$ \\
\hline Atención al proceso de envejecimiento & 1 & $1,2 \%$ \\
\hline En muchas & 2 & $2,5 \%$ \\
\hline Historia de los cuidados & 1 & $1,2 \%$ \\
\hline Ética y legislación & 1 & $1,2 \%$ \\
\hline En ninguna & 2 & $2,5 \%$ \\
\hline Prácticas & 1 & $1,2 \%$ \\
\hline Total & 79 & \\
\hline
\end{tabular}

Fuente: Elaboración propia

También se quiso saber si habían leído bibliografía específica sobre esta cuestión y se les preguntó en concreto si podían indicar algún autor/a. Las respuestas son las siguientes. 
Tabla 3

\begin{tabular}{|l|c|c|}
\hline Respuestas & Frecuencia absoluta & Porcentaje \\
\hline No he leído bibliografía específica & 28 & $71,7 \%$ \\
\hline *NC (No contesta) & 7 & $17,9 \%$ \\
\hline No lo recuerdo & 1 & $2,5 \%$ \\
\hline Artículos & 1 & $2,5 \%$ \\
\hline Libros de texto & 2 & $5 \%$ \\
\hline Total & 39 & $100 \%$ \\
\hline
\end{tabular}

Fuente: Elaboración propia

El 71,7\% no ha leído bibliografía específica sobre el tema, cabe resaltar que el $17,9 \%$ no contesten a la pregunta y el $82,1 \%$ no saben indicar ningún autor/a en referencia a la bibliografía sobre la relación sexo/género y salud.

Después del análisis realizado y a la luz de los resultados mostrados entendemos que se puede aceptar la tercera hipótesis formulada: El alumnado de tercer curso no es conocedor de las desigualdades y diferencias por razón de sexo/ género existentes actualmente en relación con el proceso salud-enfermedad.

Dado que hemos aceptado las tres subhipótesis que forman nuestro modelo de análisis podemos aceptar la hipótesis general que habíamos formulado al inicio. Es así que mantenemos que, en la formación actual de los diplomados/as enfermería, no está presente el conocimiento que existe, actualmente, sobre diferencias y desigualdades por razón de sexo/género en relación al proceso salud-enfermedad.

\section{Conclusiones}

A partir de la revisión bibliográfica realizada se muestran los conocimientos existentes sobre las desigualdades en salud por razón de sexo/género.

En los programas de las diferentes asignaturas, la utilización del genérico es importante, aunque todavía se utiliza alguna vez el masculino para referirse a los dos 
géneros y no se hace mención de una manera explicita a la relación del sexo/género con el proceso salud-enfermedad.

El alumnado tiene un conocimiento muy descriptivo en relación al tema de estudio (distribución de los tipos de cánceres por sexo, riesgo de enfermedades cardiovasculares...) y limitado a los problemas más conocidos. Consideramos que falta profundizar en los elementos que intervienen en todo el proceso de salud-enfermedad y en los problemas de salud que se estudian, puesto que el alumnado sigue señalando la gran mayoría tan sólo los factores anatomofisiològicos propios del paradigma biologista.

No hemos podido comparar nuestros resultados con estudios similares ya que no hemos encontrado publicaciones sobre este tema. Al inicio del trabajo, se hace referencia a la Ley de ordenación de las profesiones sanitarias y recordábamos que la base de la profesión de enfermería es el cuidar. En este contexto, consideramos necesario profundizar en el conocimiento actual que se tiene en relación con el sexo/género y en el proceso salud-enfermedad y trasladarlo a la disciplina de enfermería, puesto que de esta manera se garantizará la transmisión de los conocimientos necesarios para cuidar a las personas, tanto hombres como mujeres, de forma integra. Entendemos que es el momento de introducir el conocimiento existente sobre este tema en los nuevos planes de estudios y tratar la relación sexo/género y salud de una manera transversal, abriendo líneas de investigación para profundizar en como el sexo/género influye en el proceso salud-enfermedad y en los cuidados de las personas para evitar que las diferencias conlleven desigualdades en el sistema de atención a la salud. Harían falta más estudios de este tipo para reflexionar sobre el tipo de enseñanza que estamos llevando a la práctica así como para ver si se incorporan los conocimientos que se van generando a partir de los estudios realizados en la actualidad y así no perpetuar un conocimiento androcéntrico que prevalece todavía en muchos textos. 


\section{BIBLIOGRAFIA}

- Artázcoz, L. (2005): La salut des de la sensibilitat de gènere. Barcelona: Institut Català de les Dones. Quadern de l'Institut no 2.

- Bustelo, M. y Lombardo, E. (2007): Políticas de igualdad en España y en Europa. Madrid: ediciones Càtedra.

- Consejo de la Unión Europea. Diario Oficial. Conclusiones del Consejo sobre la salud de las mujeres. (2006/C146/02) 22-6-2006).

- Colectivo del Libro de Salud de las Mujeres de Boston (1982): Nuestros cuerpos, nuestras vidas. Barcelona: ICARIA.

- Collière, M. F. (1993): Promover la vida. Madrid: McGraw-Hill/Interamericana de España.

- Enhrenreich, B. y English, D. (1981): Brujas, comadronas y enfermeras. Barcelona: la Sal.

- Garcia-Moreno, C. (2000): Violencia contra la mujer. Género y equidad en la salud. Organización Panamericana de la salud y Harvard Center for population and Development Studies.

- Generalitat de Catalunya, (2003): Pla de Salut de Catalunya 2002-2005. Barcelona: Direcció General de Salut Pública.

- Generalitat de Catalunya (2005): Marcar les diferències: la representació de dones $i$ homes a la llengua. Criteris lingüístics. Barcelona: Depatament de la Presidencia.

- Instituto Nacional de Estadística (2005): Apartados consultados: Demografía y Sociedad - Salud, [en línea] Disponible en: http://www.ine.es/inebmenu/mnu_salud.htm - Izquierdo, M. J. (2003): Sistema “Sexo-género”. Curso de Formación y Acreditación en Consultoria para la igualdad de mujeres y hombres. Bloque Temático 2: Marco Técnico de la igualdad. Curso organizado por EMAKUNDE.

- Larrañaga, I.; Arregui, B. y Arpal, J. (2004): "El trabajo reproductivo o doméstico". En Gaceta Sanitaria, no. 18, pp. 31-37. 
- Lasheras, M. L.; Pires, M. y Rodriguez, M. M. (2004): Género y Salud. Instituto Andaluz de la Mujer. Junta de Andalucia.

- LEY 44/2003, de 21 de noviembre, de ordenación de las profesiones sanitarias (BOE $n^{\circ} .280$ de 22-XI-2003).

- LEY 3/2007, de 22 de marzo, para la igualdad efectiva de mujeres y hombres. (BOE $\mathrm{n}^{\mathrm{o}}$. 71 de 23-3-2007).

- LEY 121/ 000101 orgánica por la que se modifica la Ley orgánica 6/2001, de 21 de diciembre, de Universidades. (Serie A, no. 101-14, 4-4-2007).

- Martinez I. (2005): Violència: Tolerància Zero. Barcelona: Fundació "La Caixa”.

- Mateu, M. L.; Panisello, M. L.; Lasaga, A. y Bonfill, E. (2007): "Desigualdades sociales en salud de las personas mayores". En Gerokomos, nº 18, pp.30-33.

- Marrugat, J. et al. (1998) "Mortality differences between men and women following first myocardial infarction". En The Journal of the American Medical Association, oct, vol. 280, nº 16 , pp. 1405-9.

- Mazarrasa, L. (2001): "Estrategias de promoción de salud". En: Miqueo C. et al. (ed): Perspectivas de género en salud. Fundamentos científicos y socio-profesionales de diferencias sexuales no previstas. Minerva: Madrid, pp. 77-93.

- Ministerio de Sanidad y Consumo (2005): Informe salud y género 2005. Madrid: Observatorio de la mujer.

- Ministerio de Sanidad y Consumo. Instituto Nacional de estadística (2006): Encuesta Nacional de Salud. Madrid.

- Ministerio de Sanidad y Consumo. Plan de calidad para el sistema nacional de Salud (2006): Indicadores de Salud de la población española. Madrid

- Montero, I. et al. (2004): “Género y salud mental en un mundo cambiante". En Gaceta Sanitaria, $\mathrm{n}^{\circ} .18$, sup. 1, pp. 175-81.

- Navarro, V. y Quiroga, A. (2004): "Políticas de Estado de bienestar para la equidad". En Gaceta Sanitaria, n. 18, pp.147-157.

- ORDEN PRE/525/2005 de 7 de marzo. Ministerio de la Presidencia. (BOE nº. 57, del 8-3-2005).

- Organización Mundial de la Salud (2002): Integración de las perspectivas de género en la labor de la OMS. Ginebra. 
- Panisello, M. L.; Lasaga, A. y Mateu, M. L. (2000): “Cuidadores principales de ancianos con demencia que viven en su domicilio". En Gerokomos, nº. 11, pp. 174-178.

- Peiró, R. et al. (2004): "Sensibilidad de género en la formulación de planes de salud en España: lo que pudo ser y no fue”. En Gaceta Sanitaria, nº. 18, supl.2, pp. 36-46.

- Quadern CAPS (1991): Mujer y Calidad de Vida. Barcelona: CAPS.

- Rivera, M. (2003): Nombrar el mundo en femenino. Barcelona: ICARIA.

- Rohlfs, I.; Borell, C. y Fonseca, M. C. (2000): “Género, desigualdades y salud pública: conocimientos y desconocimientos”. En Gaceta Sanitaria, nº. 14, pp. 60-71.

- Rohlfs, I. et al. (2000): "La importancia de la perspectiva de género en las encuestas de salud”. En Gaceta Sanitaria, no. 14, pp. 146-155.

- Rosa-Jimenez, F. et al. (2005): “¿Solicitan las mujeres más consultas al área médica que los hombres?" En Anales de Medicina Interna, no. 11, pp. 515-519.

- Ruiz-Cantero, M. T. y Verdú-Delgado, M. (2004): "Sesgo de género en el esfuerzo terapéutico". En: Gaceta Sanitaria, n.18, pp. 118-125.

- Ruiz-Perez, I.; Blanco-Prieto, P. y Vives-Cases, C. (2004): "Violencia contra la mujer en la pareja: determinantes y respuestas sociosanitarias”. En: Gaceta Sanitaria, nº.18, pp. 4-12.

- Sau, V. (1981): Un diccionario ideológico feminista. Barcelona: Icaria. . (1993): Ser Mujer. El fin de una imagen tradicional. 2a ed. Barcelona: Icaria.

- Universitat Rovira i Virgili. Guia de centre EU Infermeria. Campus Centre. Curs 2006-2007.

- Valls-Llobet, C. (1998): “La atención de género en la Atención Primaria”. En: Quadern CAPS, n. 27, pp.46-52.

(2001): "El estado de la investigación en salud y género". En: C. Miqueo et al. (eds): Perspectivas de género en salud. Fundamentos científicos y socio-profesionales de diferencias sexuales no previstas. Minerva. Madrid, pp. 179-195. . (2006): Mujeres invisibles. $2^{\mathrm{a}}$ ed. Barcelona: debolsillo.

- Wilkinson, S. y Kitzinger, C. (1996): Mujer y Salud. Barcelona: Paidos. 